\title{
Comportamento suicida: fatores de risco e intervenções preventivas
}

\section{Suicidal behavior: risk factors and preventive interventions}

\section{Comportamiento suicida: factores de riesgo y intervenciones de prevención}

Kelly Piacheski de Abreu', Maria Alice Dias da Silva Lima", Eglê Kohlrausch"l', Joannie Fachinelli Soares ${ }^{\prime V}$

\author{
Acadêmica de Enfermagem da Universidade Federal do Rio Grande do Sul (UFRGS). Bolsista de Iniciação Científica (PIBIC). Porto Alegre, RS. E-mail: \\ kelly.piacheski@ufrgs.br \\ "Enfermeira. Doutora em Enfermagem. Professora Associada da Escola de Enfermagem (EENF) da UFRGS. Porto Alegre, RS. E-mail: malice@enf.ufrgs.br \\ III Enfermeira. Mestre em Enfermagem. Doutoranda do Programa de Pós-Graduação em Enfermagem da UFRGS. Professora Assistente da EENF/UFRGS. \\ Porto Alegre, RS. E-mail: eglek@hotmail.com \\ IV Acadêmica de Enfermagem da UFRGS. Membro do GESC. Bolsista PIBIC. Porto Alegre, RS. E-mail: joannie fachi@yahoo.com.br
}

\section{RESUMO}

As taxas de suicídio evidenciam um problema de saúde pública, e ações preventivas são necessárias. O objetivo deste artigo de atualização é discorrer sobre fatores de risco para o comportamento suicida e as possíveis intervenções utilizadas para sua prevenção na atenção primária à saúde. Os fatores de risco mais importantes para comportamento suicida são: transtornos mentais, antecedentes familiares, sexo, idade, relações familiares, abuso de substâncias, problemas físicos e situação social desfavorável. Considerando que os usuários com comportamento suicida tendem a procurar auxílio nos serviços de atenção primária antes de morrer, é possível identificar a presença desse comportamento. O foco de implementação do cuidado deve ser direcionado àqueles que se enquadram em situações de agravos que predisponham às tentativas de suicídio. Por isso, o conhecimento dos fatores de risco e intervenções preventivas para o comportamento suicida pode trazer benefícios para a comunidade e profissionais de saúde, garantindo um cuidado seguro e tecnicamente orientado. Para isso é necessária a sensibilização dos profissionais de saúde para o uso de tecnologias relacionais, bem como estratégias de acolhimento e vínculo para a identificação dos fatores de risco, bem como a estruturação de ações em saúde mental para serem desenvolvidas com suas comunidades e escolas.

Descritores: Suicídio; Tentativas de suicídio; Atenção primária à saúde; Prevenção primária.

\section{ABSTRACT}

Suicide rates evidence a public health problem so that preventive actions are needed. The objective of this updating article is discussing about risk factors that involve the suicidal behavior and the possible interventions for its prevention in the primary health care. The most important risk factors related to suicidal behavior are: mental disorders, family background, sex, age, family relationship, drugs abuse, physical problems and unfavorable social condition. Considering that users with suicidal behavior tend to look for help in the primary health care services before dying, it is possible to prevent suicide attempts. It is necessary to provide education and habilitation for health professionals, who are active in the primary health care in order to help detecting suicide risk factors. Within this sector, the focus of care implementation must be generalized and addressed to certain populations; besides, the approach must be collective and the programs developed with the purpose of comprising all of the subjects. The knowledge of risk factors and preventive interventions against suicidal behavior provides benefits for the community and health professionals as well. Besides such knowledge, investigation and follow-up of the identified cases must be carried out in primary health care.

Descriptors: Suicide; Suicide attempts; Primary health care; Primary prevention.

\section{RESUMEN}

Las tasas de suicidio demuestran un problema de salud pública, siendo necesarias acciones preventivas. El objetivo de este artículo de actualización es discurrir acerca de los factores de riesgo que envuelvan el comportamiento suicida y las posibles intervenciones para su prevención en la atención primaria de salud. Los factores de riesgo más importantes para el comportamiento suicida son: trastornos mentales, antecedentes familiares, sexo, edad, relaciones familiares, abuso de substancias, problemas físicos y condición social desfavorable. Considerando que los usuarios con comportamiento suicida tienden a buscar auxilio en los servicios de atención primaria antes de morir, es posible prevenir tentativas de suicidio. Se hace necesaria la educación y la capacitación de los profesionales de salud que actúan en la atención primaria de salud para auxiliar en la detección de factores de riesgo para el suicidio. En ese sector, el foco de la implementación del cuidado debe ser generalizado y direccionado a determinadas poblaciones; el enfoque debe ser colectivo y los programas, desarrollados con la finalidad de abarcar a todos los individuos. El conocimiento de los factores de riesgo e intervenciones preventivas para el comportamiento suicida traen beneficios para la comunidad y para los profesionales de salud. En la atención primaria de salud, además de estos conocimientos, deben ocurrir la investigación y el acompañamiento de los casos identificados.

Descriptores: Suicidio; Intento de suicidio; Atención primaria de salud; Prevención primaria. 


\section{NTRODUÇÃO}

O suicídio é um problema com várias causas. É uma ação que está ligada a fatores biológicos e psicológicos associados ao contexto sócioeconômico ${ }^{(1)}$. Entretanto, a despeito da abundante produção científica sobre o assunto, as pesquisas já realizadas parecem não estar contribuindo para a redução da incidência dos casos, pelo contrário, há uma tendência de aumento em vários países ${ }^{(2)}$

As taxas de tentativas de suicídio são, aproximadamente, dez vezes maiores que as taxas de suicídios consumados. Ou seja, aproximadamente uma em dez tentativas de suicídio termina em morte $^{(3)}$. No ano de 2000, aproximadamente 815 mil pessoas se suicidaram no mundo, ou seja, a taxa de mortalidade por suicídio é de 14,5/100.000 habitantes, representando um suicídio a cada 40 segundos ${ }^{(4)}$.

O suicídio não é um gesto com mecanismos bem esclarecidos, são diversos os fatores de risco que requerem compreensão num complexo paradigma social e comportamental. O modelo biomédico justifica o comportamento suicida como sendo o resultado final de um transtorno psiquiátrico com causas apenas biológicas. Analisa o suicídio como resultado de uma doença/injúria, com enfoque fisiopatológico. Porém, é necessário ter o conhecimento de quanto o biológico contribui para o indivíduo, sua história de vida, circunstâncias e desenvolvimento ${ }^{(5)}$.

Neste sentido, observa-se que o comportamento suicida é um tema tabu, tendo em vista a complexidade do gesto. O comportamento suicida confronta-se com o instinto de sobrevivência inerente aos humanos. É difícil compreender como alguém idealiza e planifica sua própria morte, escolhe o método que vai utilizar para isto e o ponha em prática. Possivelmente, a vontade de se aliviar de um sofrimento emocional intolerável proporciona uma aproximação do sujeito com as diversas formas de comportamento suicida.

Comportamento suicida é definido como uma ação na qual o individuo inflige-se dano (autoagressão), não importando o nível ou a razão genuína da ação. Uma definição ampla dessa forma permite que se conceitue o comportamento suicida por meio de um contínuo: os pensamentos de autodestruição, a auto-agressão, manifestada por gestos suicidas e tentativas de suicídio, e, finalmente, o próprio suicídio ${ }^{(6)}$.

Por isso o termo comportamento suicida se refere à ação de auto-agressão, bem como variáveis relacionadas às tentativas de suicídio, com alta ou baixa letalidade, que ocorrem dentro de um contexto social, e trazem elementos que indicam a procura de ajuda. É necessário levar em consideração o plano suicida, o desejo de morrer contra o de viver, e as possibilidades de alguém descobrir o plano suicida para avaliar a intensidade do comportamento suicida. A ideação suicida sem tentativa de suicídio é mais comum do que o comportamento suicida fatal ou não ${ }^{(3)}$.

O tema suicídio demanda a atenção de profissionais de diversas áreas que possam tratar dos riscos e das possibilidades de prevenção(1). É preciso levar em consideração que, atualmente, as situações sociais, como empregabilidade e desemprego, estrutura familiar, condições socioeconômicas, padrão de possibilidades de consumo de insumos, como roupas, alimentos e lazer, aceitação no meio de convivência, entre outros fatores, interagem com as predisposições biológicas para o aparecimento do comportamento suicida ${ }^{(4)}$.

Assim, uma abordagem interdisciplinar, analisando questões socioculturais e filosóficoexistenciais além dos aspectos fisiopatológicos, torna-se necessária para que se tenha uma explicação satisfatória dos motivos e causas que levam os indivíduos a tentar e consumar a autodestruição(5).

Para uma abordagem interdisciplinar para atendimento ao comportamento suicida precisa-se de uma organização do trabalho baseada no trabalho em equipe, que requer intervenções técnicas e interação entre os profissionais, que, de maneira coordenada, colocam em prática seus planos de ação. A comunicação, a articulação das ações e a compreensão das diferenças técnicas tornam-se indispensáveis para que a equipe consiga alcançar seus objetivos. Portanto, a soma dos saberes das diversas profissões envolvidas no ato de cuidar colabora para que a situação seja abordada da forma mais integral possível, e que seja garantida a preservação da vida.

Foi identificado que mais de $75 \%$ das vítimas de suicídio procuraram um serviço de atenção primária à saúde no ano de sua morte e $45 \%$ no mês que cometeram suicídio ${ }^{(6)}$. Portanto, para a prevenção do comportamento suicida, os profissionais da área de saúde pública desempenham papel fundamental na detecção precoce de fatores de risco.

Considerando as taxas nacionais e a importância das equipes da atenção básica, em 2006, o Ministério da Saúde apresentou a Estratégia Nacional para Prevenção do Suicídio, pela Portaria GM no 1.876, com a intenção de diminuir as taxas de suicídios e tentativas. Esta estratégia prevê sua implantação em parceria com a atenção básica, buscando o modelo de redes de cuidado, de base territorial e atuação transversal com outras políticas específicas, proporcionando vínculo e acolhimento. A ideia é que as equipes da Estratégia de Saúde da Família (PSF) sejam apoiadas por equipes matriciais para atender os casos de saúde mental no território de sua área $\operatorname{adscrita}^{(7)}$. 
Assim, tendo em vista a relevância do tema, o objetivo deste artigo de atualização é discorrer sobre fatores de risco para o comportamento suicida e as possíveis intervenções utilizadas para sua prevenção na atenção primária à saúde. Pretende-se contribuir para que os profissionais que se deparam com usuários com comportamento suicida possam entender melhor o gesto, ter conhecimento sobre a situação epidemiológica e planejar estratégias de prevenção.

\section{Fatores de risco para comportamento suicida}

Os fatores de risco mais importantes para comportamento suicida são: transtornos mentais os quais estão presentes em mais de $90 \%$ daqueles que cometem suicídio, antecedentes familiares, sexo, idade, relações familiares, abuso de substâncias, problemas físicos, principalmente aqueles que causam invalidez e/ou dor crônica e situação social desfavorável, como pobreza e desemprego ${ }^{(3)}$.

No Brasil, um estudo investigou as taxas de suicídio entre 1980 e 2000, fazendo uma comparação com a situação epidemiológica mundial, considerando idade e sexo. Os resultados apontaram que os índices globais de suicídio cresceram $21 \%$ em 20 anos, que os homens suicidam-se 2,3 a 4 vezes mais que as mulheres e que os idosos acima de 65 anos apresentaram as taxas mais elevadas. Os jovens entre 15 e 24 anos apresentaram o maior crescimento $^{(8)}$.

Além disso, existem diferenças entre os sexos em relação à letalidade do comportamento suicida em função das construções de gênero. As mulheres escolhem métodos menos invasivos para não perderem a beleza, enquanto os homens preferem métodos que evidenciem sua virilidade. As mulheres têm comportamento suicida com mais frequência e fazem mais tentativas, entretanto, os homens cometem mais suicídio. Essa diferença entre tentativas e atos consumados entre os sexos pode ser explicada pelo fato de que os homens, por utilizarem métodos mais letais, como armas de fogo e enforcamento, são mais efetivos nas suas tentativas de suicídio do que as mulheres, em que predominam as tentativas por envenenamento ${ }^{(9)}$.

Existem ainda, além dos fatores de risco já mencionados, problemas no nível psíquico, como ansiedade, impulsividade, transtornos de humor, transtornos afetivos, baixa auto-estima, sentimentos de desesperança e solidão, sofrimento intenso, frustrações, estresse, esquizofrenia, e psicopatologias em geral, que agravam a situação. Nesse nível destaca-se a depressão, que é fator de alto risco para suicídio, e que está cada vez mais presente na vida dos seres humanos, podendo encontrar-se desde etapas precoces da vida, como a lactância(10).

$\mathrm{Em}$ relação à percepção de sintomas depressivos, foi realizado um estudo quantiqualitativo contemplando as questões culturais, em Santa Catarina, investigando as comunidades açoriana, italiana e alemã. Os grupos evidenciaram diferenças na manifestação dos sintomas depressivos, evidenciando formas próprias de expressão desses sintomas, de acordo com a cultura(11).

Também os conflitos familiares se destacam como importante fator de risco para o suicídio. Problemas como dificuldades de relacionamento e de comunicação, ausência de afeto e falta de apoio familiar por vezes estão na origem de comportamentos suicidas. A disfuncionalidade familiar é um risco ainda maior para crianças e adolescentes, visto que as relações familiares são importantes no desenvolvimento do indivíduo ${ }^{(1)}$. Em contrapartida, o ato suicida ocasiona desajuste no funcionamento familiar, visto que os outros membros da família fazem uma avaliação dos seus atos, podendo gerar um sentimento de culpa(12). Ademais, a presença de familiares que tentaram ou cometeram suicídio é um fator agravante para o comportamento suicida. Estas informações ressaltam que a família funcional é um fator de proteção aos agravos em saúde mental, e, portanto, as ações dos profissionais de saúde precisam ser direcionadas não somente ao indivíduo, mas também ao grupo familiar.

O comportamento suicida pode ter origens genéticas. Há evidência crescente de que os fatores genéticos influenciam a predisposição ao suicídio, visto que a hereditariedade do comportamento suicida é comparável à hereditariedade de transtornos psiquiátricos, como transtorno do humor e esquizofrenia ${ }^{(3)}$.

Evidencia-se que o risco para comportamento suicida é uma conjugação entre o biológico e o psicossocial, um potencializando o outro. Dessa forma, as ações de prevenção devem ser realizadas contemplando a atenção integral ao indivíduo. O exercício de solidariedade, juntamente com as condições de adaptação do indivíduo ao sofrimento psíquico, é um fator relevante ao tratar usuários com comportamento suicida.

A integralidade do cuidado em saúde mental deveria ser entendida não apenas como uma diretriz do Sistema Único de Saúde (SUS), mas como um compromisso dos profissionais com os usuários. Essa ideia remete ao fato de que estratégias relacionais, como acolhimento e vínculo, são fundamentais para que os profissionais possam identificar, se aproximar e intervir de forma resolutiva com os usuários com comportamento suicida.

Além dos fatores de risco para o suicídio, existem fatores protetores contra o suicídio, como morar com outras pessoas, ter religião, filhos e/ou companheiros $^{(13)}$. 


\section{Intervenções para}

prevenção

do

O usuário com comportamento suicida apresenta três características principais: ambivalência, impulsividade e rigidez. O profissional de saúde deve encontrar alguma forma de aumentar o desejo pela vida, fornecendo, se possível, auxílio no momento do impulso suicida. O estabelecimento de um contrato de não suicídio entre o profissional, usuário e família pode ser firmado, visando à manutenção da vida ${ }^{(14)}$.

Visto que o suicídio é um ato complexo e multifatorial, cada caso deve receber intervenção específica. Um evento estressor pode gerar comportamento suicida, neste caso a intervenção indicada é a realização de acompanhamento desses indivíduos com risco para suicídio, desmistificando o significado que o ato representa para o usuário ${ }^{(3,15)}$.

Entende-se que saúde mental engloba um conjunto de requisitos, e não se reduz à ausência de psicopatologias. Para que a condição de saúde seja mantida são necessários outros fatores, como condições sócio-econômicas favoráveis que permitam ao indivíduo o acesso a necessidades básicas, como moradia, alimentação, lazer, cultura e educação.

Considerando que os usuários com comportamento suicida tendem a procurar auxílio nos serviços de atenção primária antes de morrer, é possível prevenir tentativas de suicídio. Para tanto, faz-se necessária a educação e a capacitação de profissionais que atuam na atenção primária à saúde para auxiliar na detecção de fatores de risco para suicídio, principalmente a depressão, prevenindo tentativas de suicídio ${ }^{(14)}$

Apesar de o transtorno depressivo representar importante fator de risco para o problema, um estudo recente demonstrou que médicos que atuam na atenção primária à saúde questionam apenas metade dos usuários com depressão sobre o comportamento suicida ${ }^{(16)}$.

Ações que auxiliam na prevenção do comportamento suicida visam à promoção da saúde mental, como grupos de auto-ajuda e a criação de condições psicossociais que estimulem a participação da comunidade por meio de atividades educativas que integrem socialmente os indivíduos, promovendo um estilo de vida saudável ${ }^{(17)}$.

Um estudo explorou características dos suicídios que ocorreram no município de Teresina (Piauí), com o intuito de subsidiar os profissionais de saúde em busca da diminuição das taxas de suicídio. Para tanto, equipes da atenção primária que trabalham inseridas na comunidade e dividem a área de abrangência em microáreas, podem gerar informações que servem como base para intervenções que são imprescindíveis no processo preventivo $^{(9)}$.

A equipe de enfermagem, principalmente na atenção primária à saúde, possui bom vínculo com a comunidade, o que possibilita a identificação de fatores de risco para o suicídio e, conseqüentemente, sua prevenção. A atuação desses profissionais tem a finalidade de promover mudanças no estilo de vida, analisar o ambiente onde o usuário encontra-se inserido, procurar fatores de risco, ajudar na identificação e tratamento de injúrias que possam trazer complicações e auxiliar na inserção dos usuários que tentaram o suicídio na comunidade(18). As enfermeiras consideram os agentes comunitários de saúde, que não pertencem à equipe de enfermagem, mas que, em geral, ficam sob supervisão delas, são os maiores responsáveis pela identificação do comportamento suicida, dada sua proximidade com a comunidade em que trabalham ${ }^{(19)}$

$\mathrm{Na}$ atenção primária à saúde, o foco de implementação do cuidado deve ser direcionado àqueles que possam ser incluídos em situações de agravos que predisponham às tentativas de suicídio. Para a prevenção do comportamento suicida são necessárias ações como a realização de programas em escolas e na comunidade. A redução do consumo e abuso de álcool e drogas entre jovens, programas que visem à redução da violência entre homens de 25 a 55 anos e remoção de barreiras que dificultam o acesso da população à saúde mental são exemplos de ações que atuariam de forma positiva para a redução dos índices de comportamento suicida ${ }^{(20)}$.

Os dados epidemiológicos indicam que as mulheres e idosos procuram mais a atenção primária à saúde do que homens jovens antes de cometerem suicídio. Dessa maneira, a prevenção do comportamento suicida deve envolver a atenção primária à saúde com intervenções para prevenir o suicídio em idosos e mulheres. Uma forma alternativa para prevenção do suicídio em homens jovens com risco para suicídio deve ser criada, visto que homens jovens procuram menos os serviços de saúde ${ }^{(6)}$.

Ocorreu, em 2004, na Áustria, um encontro com 15 especialistas no tema suicídio. Nesse evento foram identificadas cinco áreas de intervenções preventivas: programas educacionais e de conscientização para o público em geral e para profissionais, identificação dos possíveis métodos de tentativas, tratamento das desordens mentais, restrição ao acesso a meios letais e o enfoque das reportagens que aparecem na mídia sobre suicídio(16).

Sabe-se que transtornos mentais ocasionam comportamento suicida. Programas educativos e de conscientização para profissionais de saúde, comunidade e cuidadores de usuários com comportamento suicida seriam eficazes para prevenir tentativas de suicídio somente se associados ao acompanhamento contínuo desses indivíduos, bem como ao tratamento psicoterápico e farmacológico. O acesso aos possíveis meios letais deve ser restringido, e o planejamento de métodos suicidas 
precisa ser deve investigado(7,16). Portanto, os profissionais da atenção básica precisam se apropriar de estratégias que os tornem próximos dos usuários, para que estes tenham uma relação de confiança e vínculo que permita expressar seu desejo de suicídio, bem como manifestar de que forma pensam em colocar isso em prática. Além disso, a forma que determina de que maneira serão relatados casos de suicídio na mídia é importante para evitar a imitação do gesto ${ }^{(16)}$.

\section{CONSI DERAÇÕES FI NAIS}

O comportamento suicida constitui-se em preocupação para a sociedade como um todo e, em especial, para os profissionais de saúde.

Nos serviços de saúde, a atuação das equipes na identificação de fatores de risco e a utilização de estratégias preventivas para esta condição podem proporcionar mais segurança e confiança nos indivíduos com este agravo e suas famílias. No âmbito da atenção primária à saúde evidencia-se ainda mais a possibilidade de realização de intervenções preventivas.

Os profissionais de saúde, nesse nível de atenção, podem tornar-se aptos para realizar intervenções seguras, tecnicamente fundamentadas, que estimulem a proteção à vida. Isso implica em proximidade com os indivíduos e suas famílias, atenção voltada para a prevenção e habilidade para exercer suas funções profissionais, pelo fato de estarem na mesma área em que convivem as pessoas que são atendidas.

Portanto, as práticas na atenção primária à saúde precisam contemplar ações com objetivo de prevenir o comportamento suicida. Para planejamento e implementação dessas ações, há necessidade de que existam, além do conhecimento sobre os fatores de risco, a estruturação e a sistematização de formas de investigação desses fatores e o acompanhamento dos casos identificados, bem como a necessidade de que os profissionais das equipes de saúde tenham as informações pertinentes sobre a realidade de saúde da população que atendem.

No entanto, mesmo considerando a forma de organização e planejamento das ações na atenção básica, estruturadas de acordo com territorialidade e adscrição, nem sempre as equipes de saúde sentemse preparadas para intervir em relação ao comportamento suicida, possivelmente por acharem que esse problema é para os especialistas.

Neste sentido, a composição interdisciplinar da equipe é fundamental para que, mesmo sem especialistas, vários saberes se complementem para o atendimento ao comportamento suicida. A visão própria que cada disciplina tem, agregada a outras, contribui para a integralidade do atendimento.
Mesmo que os profissionais de saúde da atenção básica estejam em contato direto com os indivíduos, em sua própria comunidade, com oportunidade de acesso sobre as condições de saúde em geral, e, neste caso específico, sobre o comportamento suicida, existem muitas dificuldades.

A formação de uma rede de atenção, onde se incluem os Centros de Atenção Psicossocial, os Residenciais Terapêuticos, as Oficinas de Geração de Renda, dentro outros equipamentos, que poderiam dar suporte para as equipes da atenção básica, ainda é muito tênue, tendo mais espaços do que pontos de intersecção, fato que complica o acompanhamento fora da unidade de saúde.

Além disso, a estratégia de matriciamento das equipes da atenção básica encontra-se em um processo de implantação incipiente, e as equipes estão pouco sensibilizadas para as tecnologias relacionais, como o acolhimento e vínculo, que proporcionam uma maior aproximação e responsabilização com a população atendida. E, ainda, seria necessário que as equipes da atenção básica estruturassem ações em saúde mental para serem desenvolvidas com suas comunidades, não só no sentido da identificação de psicopatologias, mas na direção da promoção da saúde, levando-se em conta a esfera da micropolítica de saúde.

$E$, ainda, os órgãos governamentais municipais poderiam colaborar, colocando efetivamente em prática a Política Nacional de Saúde Mental e as metas da Estratégia Nacional de Prevenção do Suicídio, em nível da macropolítica de saúde.

Certamente a tarefa de prevenir o comportamento suicida não é fácil, muitas vezes frustra o profissional da atenção básica, que se sente despreparado e sem ter a quem recorrer para discutir as situações encontradas.

No entanto, é um problema que está posto, e representa um olhar para além da questão biológica, sendo necessário melhorar a qualidade de vida e considerar as possibilidades de realização de cada um, levando em conta as diferenças existentes entre os usuários e respeitando as condições e potencialidades individuais.

\section{REFERÊNCI AS}

1. Minayo MCS, Cavalcante FG, Souza ER. Methodological proposal for studying suicide as a complex phenomenon. Cad Saude Publica. 2006; 22(8): 1587-96.

2. Meneghel SN, Victora CG, Faria NMX, Carvalho LA, Falk JW. Características epidemiológicas do suicídio no Rio Grande do Sul. Rev Saude Publica. 2004; 38(6): 804- 10.

3. Mann JJ. A current perspective of suicide and attempted suicide. Ann Intern Med. 2002; 136(4): 302-11. 
4. Organização Mundial da Saúde. Relatório mundial sobre violência e saúde. Genebra: OMS; 2002.

5. Souza ER, Minayo, MCS, Malaquias JV. Suicídio de jovens nas principais capitais do Brasil. Cad Saude Publica. 2002; 18(3): 673-83.

6. Luoma JB, Martin CE, Pearson JL. Contact with mental health and primary care providers before suicide: a review of the evidence. Am J Psychiatry. 2002; 159(6) : 909-16.

7. Ministério da Saúde. Estratégia Nacional de Prevenção ao Suicídio. Brasília: Ministério da Saúde; 2006.

8. Mello-Santos C, Bertolote JM, Wang Y. ANG, Y. Epidemilogy of suicide in Brasil (1980-2000): characterization of age rates of suicide. Rev Bras Psiquiatr. 2005;27(2): 131-4.

09. Parente ACM, Soares RB, Araújo ARF, Cavalcante IS, Monteiro CFS. Caracterização dos casos de suicidio em uma capital do Nordeste Brasileiro. Rev Bras Enferm. 2007;60(4):377-81.

10. Morett AE, López BA. Suicidio, homicidio y drogadicción en niños y adolescentes. Revista médica del Hospital General de México. 1999;62(3): 183-190. 11. Lin J, Peruchi MM, Souza LH, Furlanetto LM, Langdon EJ. Percepção e expressão de síntomas depressivos em três grupos culturais catarinenses: açorianos, italianos e alemães. J. bras. psiquiatr. 2008; 57(1): 2-8.

12. Jiménez $A M$, Disotuar IM, Silveira MM, Aleaga MA. Comportamiento de la conducta suicida infantojuvenil. Rev Cubana Med Gen Integr. 1998; 14(6): 554-9.

13. Botega NJ, Barros MBA, Oliveira HB, Dalgalarrondo $\mathrm{P}$, Marín-León L. Suicidal behavior in the community: prevalence and factors associated with suicidal ideation. Rev Bras Psiquiatr. 2005; 27(1): 45-53.

14. Organização Mundial da Saúde. Preventing Suicide: a resource for Primary Health Care workers. Genebra: OMS; 2000.

15. Mann JJ, Apter A, Bertolote J, Beautrais A, Currier $D$, Haas $A$, et al. Suicide prevention strategies: a systematic review. J Am Med Inform Assoc. 2005; 294(16): 2064-74.

16. Feldman MD, Franks P, Duberstein PR, Vannoy S, Epstein R, Kravitz RL. Let's not talk about it: suicide inquirí in primary care. Ann Fam Med. 2007; 5(5): 412-18.

17. Reyes WG. La promocion de salud ante el suicídio. Rev Cubana Med Gen Integr. 2002; 18(1): 33-45.

18. Trebejo AL, Trebejo LAL. Comportamento del suicidio en ciudad de la Habana. Intervencion de enfermeria en la atencion primaria de salud. Rev Cubana Enferm. 2000; 16(2): 78-87.

19. Abreu KP, Kohlrausch ER, Lima MADS. Atendimento ao usuário com comportamento suicida: a visão dos agentes comunitarios de saúde - estudo qualitativo. Online Braz J Nurs. 2008; 7(3): 1238-50.

20. Knox KL, Conwell Y, Caine ED. If suicide is a public health problem, what are we doing to prevent it? Am J Public Health. 2004; 94(1):37-45.

Artigo recebido em 22.10.08.

Aprovado para publicação em 22.10.09.

Artigo publicado em 31.03.10. 